\section{Hepatotoxicidad a consecuencia del consumo de fluoruro en ratas albinas de la raza Holtzman.}

Flores-Ezcurra $J^{1, a}$, Flores-Ezcurra $C^{1, b}$, Loza-Suarez $J^{1, b}$. Hepatotoxicidad a consecuencia del consumo de fluoruro en ratas albinas de la raza holtzman. Rev Estomatol Herediana. 2013 EneMar;23(1):18-23.

\section{RESUMEN.}

Objetivos: Evaluar el efecto del consumo sostenido de fluoruro, sobre la estructura y función del hígado, de ratas albinas raza Holtzman. Materiales y Métodos: Estudio experimental, prospectivo y comparativo, conformado por 40 ratas hembras, de 02 meses de edad con un peso aproximado de $\mathbf{2 0 0}$ gramos, que fueron divididas al azar en $\mathbf{4}$ grupos iguales; un control y tres experimentales (I, II, III). Las ratas de los grupos experimentales recibieron diariamente fluoruro, diluido en agua para beber, con una concentración de 22,5; 45 y $135 \mathrm{mg} / \mathrm{L}$ respectivamente, durante 12 semanas. Resultados: A nivel macroscópico existió incremento del peso y volúmen hepático en los tres grupos experimentales. A nivel bioquímico se halló reducción de la albúmina sérica y prolongación del tiempo de protrombina en los tres grupos experimentales, siendo en todos significativo $(\mathrm{p}<0,05)$, donde la severidad fue mayor a medida que la dosis fue más alta. El examen microscópico reveló grumos intracitoplasmáticos, edema intracelular, escasa definición de membrana celular, degeneración balonizante, necrosis de hepatocitos, congestión vascular a predominio periportal, dilatación de la vena centrolobulillar y de los sinusoides. Los efectos fueron más notables a medida que aumentaba la concentración de fluoruro. Estos cambios no se observaron en el grupo control. Conclusiones: El consumo sostenido de fluoruro, produce a nivel del hígado, lesión hepatocelular, desde edema hasta necrosis celular, alterando la función sintética, evidenciándose con hipoalbuminemia y prolongación del tiempo de protrombina.

Palabras clave: FLUORUROS, RATAS, HEPATOTOXICIDAD.

Hepatotoxicity resulting from fluoride consumption in albino holtzman rats.

\section{ABSTRACT}

Objectives: To evaluate the effect of sustained fluoride consumption on the structure and function of the liver, albino Holtzman rats. Materials and methods: Experimental, prospective, comparative, consisting of 40 female rats two months of age with an average weight of 200 grams, were randomly divided into 4 equal groups: one control and here experimental groups (I, II, III). The rats of the experimental groups received daily fluoride diluted in drinking water at a concentrations of 22,5; 45 and $135 \mathrm{mg} / \mathrm{L}$ respectively, for 12 weeks. Results: At the macroscopic level, there was an increase of weight and liver volume in the three experimental groups. At the biochemical level was found, the reduction of serum albumin, and prothrombin time prolonged in the threeexperimental groups, being in all significant $(p<0,05)$, where the severity was increased as the dose was higher. Microscopic examination revealed intracytoplasmic clumps, intracellular edema, poor definition of the cell membrane, ballooning degeneration, hepatocyte necrosis, periportal vascular congestion, dilated lobular central vein and sinusoids. The effects were more evident with increasing fluoride concentration. These changes were not observed in the control group. Conclusions: Sustainable fluoride consumption causes heptocellular injury from edema to cell necrosis, altering the synthetic function, evidence with hypoalbuminemia and prolonged prothrombin time.

Key Words: FLUORIDES, RATS, HEPATOTOXICITY.

\section{Jimmy Flores Ezcurra ${ }^{1, a}$ Carlos Flores Ezcurra ${ }^{1, b}$ Jacqueline Loza Suarez ${ }^{1, b}$}

Universidad Inca Garcilaso de la Vega. Lima, Perú.

a Cirujano Dentista. Diplomado en Cirugía Oral.

\section{Cirujano Dentista.}

\section{Correspondencia:}

Jimmy César Flores Ezcurra

Av. San Martín 289 Dpto. 101. Pueblo Libre. Lima, Perú.

Teléfono: 511-7934644 Celular 964367107 E-mail: jflores.ezcurra@gmail.com

\section{Introducción}

El descubrimiento de las propiedades anticariogénicas del fluoruro, constituyó uno de los pasos más importantes en el desarrollo de la odontología, una vez que posibilitó el desenvolvimiento de medidas eficaces para prevenir y controlar la caries dental. Actualmente el fluoruro, es administrado terapéuticamente, mediante dos maneras; por vía sistémica, absorbida vía tracto gastrointestinal y la vía local o tópica, a nivel de la cavidad bucal. Dentro de la vía sistémica tenemos: la fluorización del agua, la leche y la sal. Dentro de la vía tópica se encuentran las pastas dentífricas, colutorios, geles y barnices fluorados (1).

Las ventajas del uso del fluoruro han sido extensamente estudiadas, pero también, son motivo de preocupación los efectos tóxicos cuando se consumen dosis por encima de las seguras y de manera prolongada. Así pues, de los productos tópicos, los dentífricos y los colutorios fluorados, han despertado interés en cuanto a la posibilidad de intoxicación y sus consecuencias (2,3). Estudios demuestran que cuando un niño utiliza pastas y/o coluto- 
rios fluorados, podría ingerir inadvertidamente dosis por encima de las consideradas seguras y experimentar un aumento no deseable de fluoruro en sangre, donde el aporte a la sangre será aun mayor y más riesgoso, si está expuesto a otras fuentes de flúor (4-7). Así mismo, son los niños quienes tienen mayor riesgo de padecer fluorosis, si tenemos en consideración el peso, la capacidad metabólica y el estado de maduración de sus huesos, siendo estos factores que acelerarían el depósito del ion flúor en el tejido óseo $(8,9)$. La base biológica, reside en el conocimiento del metabolismo del fluoruro, ya que una vez ingerido, es absorbido en un $75 \%$ a $90 \%$ en la mucosa gástrica e intestinal, por un proceso pasivo, en forma de ácido fluorhídrico $(10,11)$. De la cantidad absorbida aproximadamente el 40 a $50 \%$ se excreta por filtración glomerular en las 24 horas siguientes, un mínimo porcentaje se elimina por medio de las heces, sudor, lágrimas y saliva, el $50 \%$ restante se deposita en los tejidos calcificados (95\% tejido óseo). La cantidad acumulada dependerá del volumen ingerido, la duración de la exposición y grado de mineralización de los huesos $(12,13)$.

La incorporación del fluoruro en el tejido óseo, se realiza a manera de cristales de fluorapatita, mediante tres mecanismos: intercambio del ion flúor por iones hidroxilos y bicarbonato de las apatitas biológicas, inclusión de fluoruro en la matriz colágena durante la formación de cristales de hidroxiapatita y por último en la remodelación ósea. Ésta última, que no se detiene nunca, explica el equilibrio dinámico que se establece entre la concentración de fluoruro en los líquidos corporales (sangre) y el que se deposi- ta en el tejido óseo, este equilibrio consiste, que en cada ciclo de remodelación, se liberan constituyentes óseos a manera de iones, uno de ellos el ion flúor hacia la sangre, y desde ésta, hacia el hueso en la etapa de depósito, permitiendo no solo que el fluoruro se deposite cada vez más, a medida que es ingerido a través del tiempo, sino también, que circule constantemente en la sangre. Esto se comprueba porque en adultos expuestos durante toda su vida al fluoruro, su fluoremia basal es mayor que en los que no consumen flúor $(14,15)$.

La lista de efectos adversos no está de ninguna manera concluida, ciertamente la fluorosis dental y esquelética, representan el efecto tóxico en las células del diente y tejido óseo, pero no son las únicas células susceptibles a daño, pudiendo verse afectadas también: las células del riñón, de la mucosa gástrica, del cerebro y de manera particular del hígado, que es especialmente susceptible a sufrir daños por ser sitio activo de metabolismo y desintoxicación de sustancias endógenas y exógenas.

El objetivo del presente fue evaluar el efecto del consumo sostenido de fluoruro sobre la estructura y función del hígado en ratas albinas Holtzman, simulando la elevación de fluoruro en sangre, que se produce en la ingesta constante de flúor en los humanos, ya sea por indicación terapéutica y/o inadvertida.

\section{Material y Métodos}

Se realizó un estudio experimental, prospectivo y comparativo. Se utilizaron 40 ratas albinas hembras de la raza Holtzman de 02 meses de edad y un peso promedio de $200 \mathrm{gr}$, adquiridas del Instituto Nacional de Salud del Perú (INS), que fueron ubicadas por separado en una jaula de metal galvanizada de $15 \times 15 \times 40$ $\mathrm{cm}$, equipada con un bebedero de policarbonato y un comedero de loza para alimento, a temperatura ambiente de $24 \pm 1{ }^{\circ} \mathrm{C}$, con $60 \%$ de humedad, con 12 horas de luz y oscuridad.

Dos semanas antes del experimento propiamente dicho, las ratas solo recibieron una dieta normocalórica-proteica, Ratina ${ }^{\circledR}$ a razón de $120 \mathrm{gr} / \mathrm{Kg}$ de peso y agua destilada a voluntad. Posteriormente fueron divididas por aleatorización simple en 4 grupos de 10 animales; un grupo control y 3 grupos experimentales. Se continuó alimentando a todas las ratas con la misma dieta normocalórica-proteica, el tratamiento administrado, solo se diferenció, en la concentración de fluoruro (F-), presente en los $4 \mathrm{ml}$ de agua que consumieron diariamente los integrantes de cada grupo, durante 12 semanas. Las ratas del grupo control recibieron agua destilada sin flúor suplementario y las de los grupos experimentales I, II y III recibieron fluoruro diluido en agua destilada a concentraciones de 22,5; 45 y $135 \mathrm{mg} / \mathrm{L}$ respectivamente.

Se utilizó Fluoruro de Sodio (NaF) al 2\% (Fluofar ${ }^{\circledR}$ neutro), que tiene una concentración de F- de 9046 miligramos por litro de solución, a partir de la cual, se realizó el cálculo de diluciones (flúor/agua), las cuales fueron 1/401 para una concentración final de $22,5 \mathrm{mg} / \mathrm{L}$; $1 / 200$ para una concentración final de $45 \mathrm{mg} / \mathrm{L}$ y de 1/66 para una concentración final de $135 \mathrm{mg} / \mathrm{L}$. A continuación se realizó la certificación de concentraciones, en el departamento de Química del Centro Nacional de Alimentación del Instituto Nacional de Salud (CE- 
NAN-INS), utilizando un titulador analizador de iones automático 751 GPD Titrino, marca Metrohn, con electrodo selectivo de flúor y electrodo de referencia de $\mathrm{Ag} / \mathrm{Cl}$, ejecutándose el método potenciómetro por ion selectivo (16).

Al término del periodo experimental, previo ayuno de 14 horas, los animales fueron anestesiados por inhalación de cloroformo, extrayéndosele sangre por punción cardiaca, la cual fue trasladada en tubos de ensayo, rotulados con el código correspondiente a la rata de origen, al Servicio Académico Asistencial de Análisis Clínicos de la Facultad de Farmacia y bioquímica de la Universidad Nacional Mayor de San Marcos, donde se determinó la concentración cuantitativa de albúmina sérica y el tiempo de protrombina mediante los métodos verde de bromocresol y coagulómetro RAL Clot SP respectivamente, a continuación las ratas fueron sacrificadas y disecadas, extrayéndoseles el hígado, para luego determinarse el peso mediante una balanza digital. El volumen hepático se registró mediante el método de volumen desplazado de cloruro de sodio al $0,9 \%$, al sumergir el hígado en una probeta de vidrio. Finalmente se realizó la fijación de las muestras en formol al $10 \% \mathrm{du}-$ rante 4 días para su posterior corte y evaluación histopatológica, para la identificación de signos de daño celular, por un médico patólogo cegado. Con relación a las consideraciones éticas y en cumplimiento a los protocolos de experimentación con animales, se aplicó la eutanasia, haciendo uso de pentobarbital, en dosis de $100 \mathrm{mg} / \mathrm{Kg}$, con el fin de evitarles dolor y angustia (17).

Los datos fueron procesados utilizando el paquete estadístico SPSS versión 20 . Se consideró significa- tiva una $\mathrm{p}<0,05$ y un intervalo de confianza del 95\%. Se aplicó el método de comparación múltiple de Tukey a los valores obtenidos al administrar diferentes concentraciones.

\section{Resultados}

El peso y volumen hepático aumentó en todos los grupos que consumieron fluoruro según aumentaba la dosis. El consumo de fluoruro tuvo un efecto significativamente diferente entre el peso hepático promedio del grupo que consumió 135 $\mathrm{mg} / \mathrm{L}$ y el grupo control $(\mathrm{p}<0,05)$ (Tabla 1). En los grupos que consumieron 45 y $135 \mathrm{mg} / \mathrm{L}$, el volumen hepático fue significativamen- te mayor que en el grupo control, a medida que aumentaba la dosis de fluoruro $(\mathrm{p}<0,05)$ (Tabla 2).

A medida que la dosis de fluoruro aumentó, la concentración de albúmina sérica de los grupos I, II y III fue significativamente menor que en el grupo control $(\mathrm{p}<0,05)$ (Tabla 3).

El tiempo de protrombina de los grupos experimentales fue significativamente mayor que el grupo control, según aumentaba la dosis de fluoruro $(\mathrm{p}<0,05)$ (Tabla 4).

Los hígados del grupo control mostraron una estructura histológica regular con el patrón carac-

Tabla 1. Efecto sobre el peso del hígado, después de los tratamientos en ratas con fluoruro durante 12 semanas.

\begin{tabular}{llc}
\hline Fluoruro consumido $(\mathrm{mg} / \mathrm{L})$ & Peso hepático $(\mathrm{gr})$ & Test de Tukey \\
(n=10 por grupo) & Media $(\mathrm{X})$ & \\
\hline 0 (control) & $9,07 \pm 0,02^{*}$ & ------- \\
22,5 & $9,15 \pm 0,03^{*}$ & 0,643 \\
45 & $9,20 \pm 0,03^{*}$ & 0,219 \\
135 & $9,28 \pm 0,27^{*}$ & 0,014 \\
\hline
\end{tabular}

*Desviación estándar. \Significativa $(\mathrm{p}<0,05)$ en comparación al grupo control.

Tabla 2. Efecto sobre el volumen del hígado, después de los tratamientos en ratas con fluoruro durante 12 semanas.

\begin{tabular}{lcc}
\hline Fluoruro consumido $(\mathrm{mg} / \mathrm{L})$ & Volumen hepático $(\mathrm{ml})$ & Test de Tukey \\
$(\mathrm{n}=10$ por grupo $)$ & Media $(\mathrm{X})$ & \\
\hline 0 (control) & $11,2 \pm 0,63^{*}$ & 0,397 \\
22,5 & $11,7 \pm 0,67^{*}$ & 0,015 \\
45 & $12,2 \pm 0,42^{*}$ & \\
135 & $12,5 \pm 0,97^{*}$ & $0,001 \downarrow$ \\
\hline
\end{tabular}

*Desviación estándar. $\$ Significativa $(\mathrm{p}<0,05)$ en comparación al grupo control. 
Tabla 3. Efecto sobre la concentración de albúmina sérica, después de los tratamientos en ratas con fluoruro durante 12 semanas.

\begin{tabular}{lcc}
\hline Fluoruro consumido $(\mathrm{mg} / \mathrm{L})$ & Albúmina sérica(gr/dl) & Test de Tukey \\
$(\mathrm{n}=10$ por grupo) & Media $(\mathrm{X})$ & \\
\hline 0 (control) & $5,5 \pm 0,67^{*}$ & 0,012 \\
22,5 & $5,37 \pm 0,12^{*}$ & 0,000 \\
45 & $5,07 \pm 0,12^{*}$ & \\
135 & $4,7 \pm 0,00^{*}$ & 0,000 \\
\hline
\end{tabular}

*Desviación estándar. \ignificativa $(\mathrm{p}<0,05)$ en comparación al grupo control.

Tabla 4. Efecto sobre el tiempo de protrombina, después de los tratamientos con fluoruro durante 12 semanas.

\begin{tabular}{lcc}
\hline Fluoruro consumido $(\mathrm{mg} / \mathrm{L})$ & Tiempo de protrombina(s) & Test de Tukey \\
$(\mathrm{n}=10$ por grupo $)$ & Media $(\mathrm{X})$ & \\
\hline 0 (control) & $16,46 \pm 0,18^{*}$ & 0,000 \\
22,5 & $17,84 \pm 0,05^{*}$ & 0,000 \\
45 & $19,56 \pm 0,05^{*}$ & \\
135 & $20,70 \pm 0,11^{*}$ & 0,000 \\
\hline
\end{tabular}

*Desviación estándar. \$Significativa $(\mathrm{p}<0,05)$ en comparación al grupo control.

terístico de lóbulos hexagonales (Fig. 1). En el grupo I, en algunos campos de visión hubo edema intracelular de los hepatocitos, escasa definición de membrana celular y grumos intracitoplasmáticos (Fig. 2). El grupo II mostró degeneración balonizante, vacuolización, plegamiento de membranas celulares, necrosis de hepatocitos, dilatación de sinusoides. En el grupo III, los efectos fueron más pronunciados, la estructura lobular se mostró alterada con necrosis de hepatocitos, infiltrado inflamatorio, degeneración balonizante, dilatación de la vena centro lobulillar y de los sinusoides, congestión vascular a predominio periportal (Fig. 3). Todas estas lesiones no se observaron en el grupo control.

\section{Discusión}

La presente investigación, ha evaluado el efecto de la exposición al fluoruro, sobre la estructura y

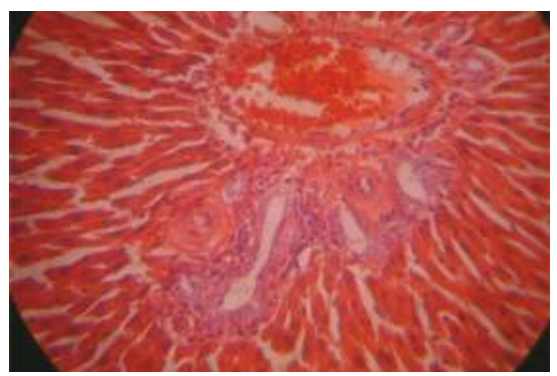

Fig. 1 Microfotografia mostrando el área portal. Grupo control. H\&E. 400X.

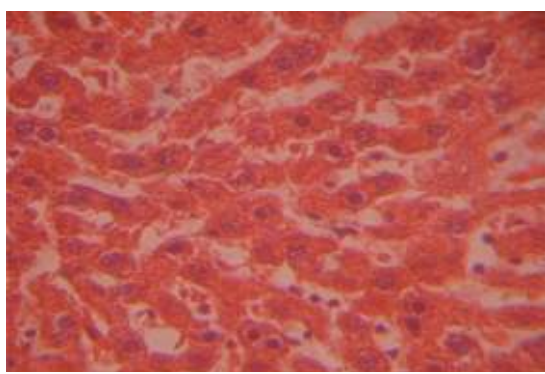

Fig. 2 Microfotografía que muestra dilatación sinusoidal, hepatocitos balonados. Grupo experimental II. H\&E. 400X.

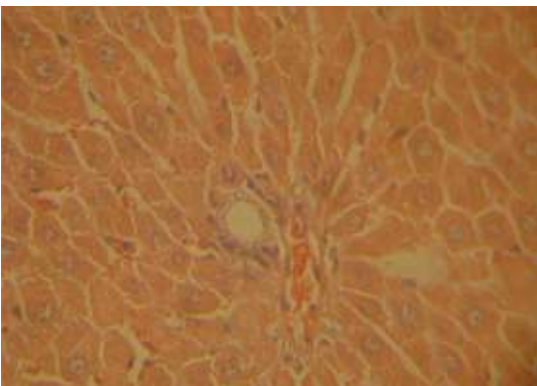

Fig. 3 Microfotografía que muestra el área periportal, se observan cambios necrobióticos, reducción del número de hepatocitos, grumos intracelulares y degeneración balonizante. Grupo experimental III. H\&E. $400 X$.

función del hígado de ratas albinas hembras, simulando la elevación de fluoruro en sangre, que se produce en el consumo sostenido de fluoruro en los humanos, ya sea por indicación terapéutica y/o inadvertida, los efectos son el resultado del equilibrio dinámico y proporcional que se establece entre el ion flúor depositado en el hueso y el liberado desde este hacia la sangre (14), para tal fin, se utilizaron concentraciones de fluoruro en el agua de bebida de 22,5; 45 y $135 \mathrm{mg} / \mathrm{L}$. Los estudios de Whitford y col. demuestran que los efectos no deseables del fluoruro son más evidentes en animales que ingirieren concentraciones a partir de $25 \mathrm{mg} / \mathrm{L}$, sugieren también, que el contenido de fluoruro consumido en agua, debe ser hasta cinco veces mayor en ratas, para conseguir un nivel de fluoruro en plasma, compatible a la que se observaría en humanos $(18,19)$. Por tanto, las concentraciones de fluoruro aquí utilizadas, equivalen a las que consumiría un ser humano, en una medida de 4,$5 ; 9$ y 27 $\mathrm{mg} / \mathrm{L}$ por día, siendo valores factibles de producirse en la vida diaria.

Concluidas las 12 semanas de exposición al flúor, se encontró incremento progresivo del peso hepático promedio de los grupos experimentales; sin embargo, solo 
fue significativo en comparación al grupo control, el grupo que consumió $135 \mathrm{mg} / \mathrm{L}$ con un $\mathrm{p}=0,014$; dato que contrasta al encontrado por Chattopadhyay y col. que no encontraron cambios significativos en el peso orgánico (20). Con respecto al volumen hepático promedio, evidenció su acrecentamiento, no obstante, solo fue significativo en comparación al grupo control, en los grupos que consumieron $45 \mathrm{mg} / \mathrm{L}(\mathrm{p}=0,015)$ y $135 \mathrm{mg} / \mathrm{L}$ $(\mathrm{p}=0,001)$, resultados que coinciden a los encontrados por Chattopadhyay y col. (20) y Dabrowska y col. (21), aunque, difieren a los observados por Camargo y col. que no encontraron cambios en el volumen hepático (22).

La medición de la concentración de la albumina sérica y tiempo de protrombina tuvo la finalidad de evaluar el efecto del fluoruro sobre la función de síntesis en el hígado, observándose un descenso significativo de la concentración de albúmina sérica promedio, en comparación al grupo control, en los grupos que consumieron $22,5 \mathrm{mg} / \mathrm{L}$ $(\mathrm{p}=0,012), 45 \mathrm{mg} / \mathrm{L}(\mathrm{p}=0,000)$ y $135 \mathrm{mg} / \mathrm{L}(\mathrm{p}=0,000)$, lo cual fue concordante a lo encontrado por Bouaziz y col. que evidenció la disminución del nivel sérico de proteínas totales, posterior a la ingesta de flúor (23). Así mismo, con respecto al tiempo de protrombina entre grupos, se encontró una clara tendencia a la prolongación del tiempo, siendo significativa en todos los grupos experimentales en comparación al grupo control. Debido a que no se encontraron antecedentes que midan esta variable, no pudo compararse con otros estudios.

Al examen anatomopatológico se advirtió la formación incipiente de grumos intracitoplasmáticos con escasa definición de membrana celular en el grupo que consumió 22,5 $\mathrm{mg} / \mathrm{L}$, convirtiéndose en lesiones mas graves a medida que aumentaba la dosis consumida, como en los grupos con dosis de 45 y $135 \mathrm{mg} / \mathrm{L}$, en los que se observó degeneración balonizante, vacuolización, plegamiento de membranas celulares, dilatación de la vena centro lobulillar y de los sinusoides hepáticos, congestión vascular a predominio periportal y necrosis hepátocelular, lesiones también descritas por Chattopadhyay y col. (20) y Dabrowska y col. (21).

El mecanismo patológico que explicaría el daño a nivel celular debido al fluoruro, es la formación de especies reactivas de oxigeno, y con ello, la alteración de la actividad de enzimas celulares como la ATPasa y la fosforilasa, enzimas que participan en la producción de energía metabólica, por lo que su inactividad ocasionaría la muerte celular $(24,25)$. No obstante para que dicho mecanismo se lleve a cabo, necesita de un nivel de fluoruro sérico elevado y de la disminución de la capacidad protectora, dado por sistemas enzimáticos anti radical libre.

En conclusión, en condiciones experimentales se ha demostrado que el consumo sostenido de fluoruro, a nivel del hígado, produce lesión hepatocelular, desde edema hasta necrosis celular, alterándose la función sintética, evidenciándose con hipoalbuminemia y prolongación del tiempo de protrombina.

\section{Agradecimientos:}

Institución donde se realizó el estudio: Universidad Nacional de San Marcos, Facultad de Medicina
Humana, Bioterio de investigación del Departamento de Ciencias Dinámicas.

Dr. Manuel Aníbal Orrego Velásquez, médico cirujano especialista en Anatomía Patológica Jefe del Departamento de Patología Clínica y Anatomía Patológica del Hospital María Auxiliadora.

CD. Enrique Gabrielli Alfaro, docente e investigador de la Universidad Nacional Federico Villareal y de la Universidad Inca Garcilaso de la Vega.

\section{Referencias Bibliográficas}

1. Cardenas D. Fundamentos de odontología: Odontología pediátrica. 3ra. Ed. Medellín: Corporación para investigaciones biológicas; 2003.

2. García JM, García F, Varela M, González A. Absorción sistémica de flúor en niños secundaria al cepillado con dentífrico fluorado. Rev Esp Salud Pública. 2009;83:415-25.

3. Barbería E, Cardenas D, Suárez M, Maroto M. Fluoruros tópicos: Revisión sobre su toxicidad. Rev Estomatol Herediana. 2005;15(1):86-92.

4. García JM, García F, González D. Pasos a vías sistémicas del flúor vehiculado en dentífricos fluorados en niños: una llamada de atención. Cient Dent. 2009;6(2):85-92.

5. Tan BS, Razak IA. Fluoride exposure from ingested toothpaste in 4-5 years old Malaysian children. Community Dent Oral Epidemiol. 2005;33(5):317-25.

6. Zuanon AC, Aranha AM. Mouthwash ingestion by preschool children. J Clin Pediatr Dent. 2005;30(1):15-7.

7. Dincer E. Why do I have spots on my front teeth? N Y State Dent J. 2008 Jan;74(1):58-60. 
8. Stookey GK. Review of fluorosis risk of self-applied topical fluorides: dentifrices, mouthrinses and gels. Community Dent Oral Epidemiol. 1994;22(3):181-6.

9. Whitford GM. Fluoride metabolism an excetion in children. J Public Health Dent. 1999;59(4):224-8.

10. Whitford GM, Williams JL. Fluoride absorption: independence from plasma fluoride levels. Proc Soc Exp Biol Med. 1986;181(4):550-4.

11. Whitford GM. Intake and metabolism of fluoride. Adv Dent Res. 1994; Jun 8(1):5-14.

12.Parkins FM, Tinanoff N, Moutinho M, Anstey MB, Waziri $\mathrm{MH}$. Relationships of human plasma fluoride and bone fluoride to age. Calcif Tissue Res. 1974;16(4):335-8.

13. Whitford GM. The metabolism and toxicity of fluoride. Monogr Oral Sci. 1996;16(2):1-153.

14. De Carvalho JG, De Oliveira RC, Buzalaf MA. Plasma as an indicator of bone fluoride level in rats chronically exposed to fluoride. J Appl Oral Sci. 2006;14(4):238-41.

15.Puche R, Rigalli A. Metabolismo del flúor. Actual Osteol. 2007;3(1):10-2.

16. Aguilar P. Validación del método potenciómetro por ion selectivo para determinación de flúor en sal, agua y orina. Rev Med Exp. 2001;18(1-2):21-3.

17. Morton DB, Griffiths PHM. Guidelines on the recognition of pain, distress and discomfort in experimental animals and a hypothesis for assessment. Vet Rec. 1985;116(16):431-6.

18.Angmar-Mansson B, Whitford GM. Plasma fluoride level and enamel fluorosis in the rat. Caries Res. 1982;16(4):334-9.

19.Angmar-Mansson B, Whitford GM. Enamel fluorosis related to plasma $\mathrm{F}$ in the rat. Caries Res. 1984;18(1):25-32.

20.Chattopadhyay A, Podder S, Agarwal S, Bhattacharya S. Fluoride-induced histopathology and synthesis of stress protein in liver and kidney of mice. Arch Toxicol. 2011;85(4):327-35

21.Dabrowska E, Letko R, Balunowska M. Effect of sodium fluoride on the morphological picture of the rat liver exposed to $\mathrm{NaF}$ in drinking water. Adv Med Sci. 2006;51:91-5.

22. Camargo AM, Merzel J. Histological and histochemical appearance of livers and kidneys of rats after long-term treatment with different concentations of sodium fluoride in drinking water. Acta Anat (Basel). 1980;108(3):288-94

23. Bouaziz H, Ketata S, Jammoussi $\mathrm{K}$, et al. Effects of sodium fluoride on hepatic toxicity in adult mice and their suckling pups. Pesticide Biochemistry and Physiology. 2006;86(3):124-30.

24. Guo X, Sung G, Sun Y. Oxidative stress from fluoride-induced hepatotoxicity in rats. Fluoride. 2003;36(1):25-9.

25.Kamisnky LS, Mahoney MC, Leach J, Melius J, Miller MJ. Fluoride: benefits and risk of exposure. Bone. 1990;14(16):17.

Recibido : 15-11-2011

Aceptado: 10-03-2012 